\title{
Flow and Heat Transfer during an Expansion Stroke in a Composite Fluid/Porous System
}

\author{
Z. Nessrine ${ }^{12}$, B. Ayda ${ }^{1}$, D. Hcen ${ }^{1}$ and B.N. Sassi ${ }^{1}$ \\ ${ }^{1}$ Laboratoire d'Etude des Systèmes Thermiques et Energétiques \\ Ecole Nationale d'Ingénieurs de Monastir,Rue Ibn Eljazzar, 5019, Monastir, Tunisie \\ ${ }^{2}$ Institut Supérieur des Mathématiques Appliquées et Informatique de Kairouan \\ Avenue Assad Iben Fourat - 3100 Kairouan \\ Email:zahi_nessrine@yahoo.fr
}

(Received January 1, 2009; accepted July 20, 2009)

\begin{abstract}
The purpose of this study is to analyze the fluid flow and heat transfer in a pipe partially filled with porous media and provided with a flat piston during an expansion stroke. In addition to the Navier-Stokes equation for the fluid region, Brinkman-Forchheimer-Lapwood-extended Darcy's model is introduced into the numerical solver to simulate flow and heat transfer in the porous insert. The heat transfer in porous media is studied by using the assumption of local thermal equilibrium. The discretization procedure is based on Control-Volume-based Finite Element Method. The coupled pressure-velocity equations are solved using the SIMPLER algorithm. This investigation concerns the hydrodynamic characteristics, using the Reynolds numbers and the porosity effects, and the heat transfer characteristics using the heat capacity and the thermal conductivity ratios effects.
\end{abstract}

Keywords: Numerical simulation, fluid flow, heat transfer, porous insert, piston driven flow

\section{NOMENCLATURE}

$\begin{array}{ll}\mathrm{C} & \text { Total piston stroke }(\mathrm{m}) \\ \mathrm{C}_{\mathrm{p}} & \begin{array}{l}\text { Specific heat capacity at constant pressure } \\ \left(\mathrm{J}^{-1} \cdot \mathrm{Kg}^{-1}\right)\end{array} \\ \mathrm{R} & \text { Cylinder radius }(\mathrm{m}) \\ \mathrm{Da} & \text { Darcy number } \\ \mathrm{k} & \text { Thermal conductivity }\left(\mathrm{W} \cdot \mathrm{m}^{-1} \cdot \mathrm{K}\right) \\ \mathrm{K} & \text { Porous medium permeability }\left(\mathrm{m}^{2}\right) \\ \mathrm{L}(\mathrm{t}) & \text { Instantaneous cylinder length }(\mathrm{m}) \\ \mathrm{L}_{\mathrm{p}} & \text { Porous region length }(\mathrm{m}) \\ \mathrm{M}_{0} & \text { Mach number } \\ \mathrm{P} & \text { Pressure }(\mathrm{Pa}) \\ \mathrm{P}_{\mathrm{m}} & \text { Space average pressure }(\mathrm{Pa}) \\ \mathrm{Pr} & \text { Prandtl number } \\ \mathfrak{R} & \text { Constant Perfect gaz }\left(\mathrm{J} \mathrm{Kg} \mathrm{Kg}^{-1} \cdot \mathrm{K}^{-1}\right) \\ \mathrm{R}_{\mathrm{c}} & \text { Heat capacity ratio } \\ \mathrm{Re} & \text { Reynolds number } \\ \mathrm{R}_{\mathrm{k}} & \text { Thermal conductivity ratio } \\ \mathrm{S}_{\mathrm{p}} & \text { Instantaneous grid velocity }\left(\mathrm{ms}^{-1}\right) \\ \mathrm{t} & \text { Time }(\mathrm{s}) \\ \mathrm{T} & \text { Temperature }(\mathrm{K})\end{array}$

$\begin{array}{ll}\mathrm{u} & \text { Axial velocity }\left(\mathrm{ms}^{-1}\right) \\ \mathrm{v} & \text { Radial velocity }\left(\mathrm{ms}^{-1}\right) \\ \mathrm{V}_{\mathrm{p}} & \text { Piston velocity }\left(\mathrm{ms}^{-1}\right) \\ \mathrm{z}, \mathrm{r} & \text { Cylindrical coordinate }(\mathrm{m}) \\ \varepsilon & \text { Porosity of porous medium } \\ \Phi & \text { General physical variable } \\ \phi & \text { Convective heat flux } \\ \Gamma & \text { Diffusion coefficient } \\ \lambda & \text { Binary parameter } \\ \Lambda & \text { Permeability ratio } \\ \mu & \text { Dynamic viscosity }\left(\mathrm{kg} \cdot \mathrm{m}^{-1} \cdot \mathrm{s}^{-1}\right) \\ \nabla & \text { nabla operator } \\ \Pi & \text { Dynamic pressure }(\mathrm{Pa}) \\ \rho & \text { Density }\left(\mathrm{kg} \cdot \mathrm{m}^{-3}\right) \\ \omega & \text { Dimensionless pulsation }\left(\mathrm{s}^{-1}\right) \\ \mathrm{f} & \text { Fluid index } \\ \mathrm{sd} & \text { Solid index } \\ \mathrm{o} & \text { Initial value of the physical variable index } \\ \infty & \text { Free stream index }\end{array}$




\section{INTRODUCTION}

Transport phenomena through porous media have been of continuing interest due to its presence in diverse engineering applications such as electronic cooling, building thermal insulations, heat exchangers and Stirling engines. In order to optimize the design considerations for various specific applications, it is necessary to better understand fundamental mechanisms in the fluid flow and heat transfer. In this thematic, several investigations were performed.

Vafai and Kim (1990) investigated the thermal performance for a composite porous medium-fluid system. Their results showed that the porous substrate can enhance the thermal performance of an external thermal boundary. This enhancement depends essentially on the thermal conductivity ratio. Hwang and Chao (1992) studied experimentally the thermal performance of a porous channel. Their results illustrated that the thermal enhancement could be obtained by using a high porous medium thermal conductivity. A numerical study of a porous block mounted on a heated wall in a laminar flow channel to enhance convection heat transfer rate was demonstrated by Fu et al. (1996). All the non-Darcian effects are considered. The results indicate that the thermal performances are enhanced by using a porous block with higher porosity and higher Darcy umber. Recently, Dhahri et al. (2006) performed a numerical study of forced convection in a pipe filled with a porous medium and subjected to a laminar reciprocating flow of air. Their results showed that the time-space-averaged Nusselt number decreases with the thermophysical properties and increases with the Darcy number. Thus, porous inserts continue to be an attractive choice in heat transfer enhancement.

Limited data exist for the case where the porous insert is arranged in a close geometry and provided with a piston. Indeed, the transient and macro response of piston-driven flow in pipe partially filled with porous media were investigated experimentally by Park et al. (2002) and Nika et al. (2004), and numerically by Hong et al. (2002) and Boucher et al. (2007) to improve the thermal performance of Stirling engine without giving consideration to the basic aero-thermodynamics phenomena. Recently, Zahi et al. (2008) presented a baseline framework for predicting the piston-driven flow features in a confined pipe partially filled with porous media during a compression stroke.

This study proposes an extended application of the previous study (Zahi et al. 2008) to explain the dynamic and the thermal behavior of the fluid flow during the expansion stroke and through the porous layer. The Brinkman-Lapwood-Forchheimer-extended Darcy model with a constant porosity is employed for the porous medium region. The porous insert is heated with constant wall temperature condition. The effects of the Reynolds number and the porosity are presented and discussed in terms of stream function, isotherms, convective heat flux and Nusselt number. The extensive heat transfer analysis is performed in terms of the thermal conductivity ratio, and the heat capacity ratio effects.

\section{Problem Formulation}

The physical problem is shown in Fig. 1. This diagram displays a 2D axisymmetric, compressible, laminar and subsonic flow in a cylinder partially filled with a porous media and provided with a flat piston. The porous medium is considered to be homogeneous, isotropic and in local thermal equilibrium (LTE) with saturated fluid. The flow over the fluid region is governed by the compressible Navier-Stokes equations using the Brinkman-Forchheimer-Lapwood-Extended Darcy model in the porous region. The governing conservation equations for the present problem can be written separately for the fluid region and the porous region. These equations were solved using a unified onedomain approach. The advantages of such a formulation are the ensuring of the interfacial conditions satisfaction and the elimination of the inner iteration loops at the interfaces. Consequently, the two sets of equations for the fluid and the porous regions are combined into one set by introducing a binary parameter $\lambda$ :

$$
\lambda= \begin{cases}1 & \text { in the porous region } \\ 0 & \text { in the fluid region }\end{cases}
$$

We make the above equations dimensionless using the following scales: $\mathrm{D}$ for length, $\mathrm{V}_{\mathrm{p}}$ for velocity, $\mathrm{T}_{0}$ for temperature and $\rho_{0}$ for density. The total pressure can be decomposed into a dynamic pressure $\Pi$ and a space averaged pressure (thermodynamic pressure) $\mathrm{P}_{\mathrm{m}}$ : $p(r, z, t)=p_{m}(t)+\Pi(r, z, t)$. The mean pressure is normalized by the initial pressure $\mathrm{p}_{\mathrm{m} 0}$, defined by the perfect gaz equation: $p_{m 0}=\mathfrak{R} \rho_{0} T_{0}$, and the dynamic pressure by $\rho_{0} V_{p}^{2}$, the last equation becomes in nonadimensional formulation:

$p(r, z, t)=p_{m}(t)+\gamma M_{0}^{2} \Pi(r, z, t)$

where $M_{0}$ denotes a reference Mach number and $\gamma=C_{p} / C_{v}$. The resulting dimensionless equations are shown below:

Continuity equation:

$$
\frac{\partial \rho}{\partial t}+\frac{\partial \rho u}{\partial z}+\frac{1}{r} \frac{\partial(\rho r v)}{\partial r}=0
$$

Momentum equation in $\mathrm{z}$ direction:

$$
\begin{aligned}
& \frac{\partial \rho u}{\partial t}+\frac{\partial}{\partial z}\left[\left(\lambda\left(\frac{1}{\varepsilon}-1\right)+1\right) \rho u u\right] \\
& +\frac{1}{r} \frac{\partial}{\partial r}\left[\left(\lambda\left(\frac{1}{\varepsilon}-1\right)+1\right) r \rho u v\right]=-\frac{\partial \Pi}{\partial z} \\
& +\frac{1}{\operatorname{Re}}\left[\frac{\partial^{2} u}{\partial z^{2}}+\frac{1}{r} \frac{\partial}{\partial r}\left(r \frac{\partial u}{\partial r}\right)\right] \\
& -\lambda\left[\frac{\varepsilon \Lambda}{D a \operatorname{Re}}+\frac{F \varepsilon \Lambda^{1 / 2}}{\sqrt{D a}}\|\vec{u}\| \rho\right] u
\end{aligned}
$$


Momentum equation in $\mathrm{r}$ direction:

$$
\begin{aligned}
& \frac{\partial \rho v}{\partial t}+\frac{\partial}{\partial z}\left[\left(\lambda\left(\frac{1}{\varepsilon}-1\right)+1\right) \rho v u\right] \\
& +\frac{1}{r} \frac{\partial}{\partial r}\left[\left(\lambda\left(\frac{1}{\varepsilon}-1\right)+1\right) r \rho v v\right]=-\frac{\partial \Pi}{\partial z} \\
& +\frac{1}{\operatorname{Re}}\left[\frac{\partial^{2} v}{\partial z^{2}}+\frac{1}{r} \frac{\partial}{\partial r}\left(r \frac{\partial v}{\partial r}\right)\right] \\
& -\lambda\left[\frac{\varepsilon \Lambda}{D a \operatorname{Re}}+\frac{F \varepsilon \Lambda^{1 / 2}}{\sqrt{D a}}\|\vec{u}\| \rho\right] v
\end{aligned}
$$

Energy equation:

$$
\begin{aligned}
& \frac{\partial\left(\lambda\left((\varepsilon-1) \rho+(1-\varepsilon) R_{c}\right)+\rho\right) T}{\partial t} \\
& +\left[\frac{\partial(\rho u T)}{\partial z}+\frac{1}{r} \frac{\partial}{\partial r}(r \rho v T)\right]=\frac{\gamma-1}{\gamma} \frac{\partial P_{m}}{\partial t} \\
& +\frac{1}{\operatorname{PrRe}}\left[\frac{\partial^{2}}{\partial z^{2}}\left[\lambda\left((\varepsilon-1)+(1-\varepsilon) R_{k}\right)+1\right] T\right] \\
& \left.+\frac{1}{\operatorname{PrRe}}\left[\frac{1}{r} \frac{\partial}{\partial r}\left(\eta \lambda\left((\varepsilon-1)+(1-\varepsilon) R_{k}\right)+1\right] \frac{\partial T}{\partial r}\right)\right]
\end{aligned}
$$

The dimensionless quantities appearing in the above equations are defined as shown below:

$$
\begin{aligned}
& \operatorname{Re}=\frac{\rho D V_{p}}{\mu}, D a=\frac{K_{\infty}}{D^{2}}, \operatorname{Pr}=\frac{\mu_{f} C p_{f}}{k_{f}}, \\
& R_{k}=\frac{k_{s d}}{k_{f}}, R_{c}=\frac{\left(\rho_{0} C\right)_{s d}}{\left(\rho_{0} C\right)_{f}} \text { and } \Lambda=\frac{K_{\infty}}{K} .
\end{aligned}
$$

The geometric function $\mathrm{F}$ and the permeability $K$ of the porous medium are based on Ergun's (1952) model and are expressed as in Vafai (1984) investigation:

$$
K=\frac{\varepsilon^{3} d_{p}^{2}}{150(1-\varepsilon)^{2}} \text { and } F=\frac{1.75}{\sqrt{150 \varepsilon^{3}}}
$$

where $\varepsilon$ and $\mathrm{d}_{\mathrm{p}}$ are, respectively, the porosity and the particle diameter.

The flow being compressible, we only take account of the temporal variation of the density and we discount its spatial variation since we are under the hypothesis of weak Mach number $\left(\mathrm{M}_{0} \prec 10 \%\right)$. The expression of the density variation is given by:

$$
\rho(t)=\rho\left(t_{0}\right) \frac{\left[V_{1}\left(t_{0}\right)+V_{3}+\varepsilon V_{2}\right]}{\left[V_{1}(t)+V_{3}+\varepsilon V_{2}\right]}
$$

$t$ and $t_{0}$ are the new and old time levels respectively. $V_{i}$ is the corresponding volume to the $\mathrm{i}$ region.
Fluid in the domain is initially at rest, the initial temperature and density fields are assumed to be uniform and constant $\left(T_{0}\right.$ and $\left.\rho_{0}\right)$.

The boundary conditions are specified as follows:

On the moving plate $(\mathrm{z}=\mathrm{L}(\mathrm{t}), 0<\mathrm{r}<\mathrm{R})$ :

$\left\{\begin{array}{l}u=v_{p}(t)=V_{p} \sin \left(\omega_{p} t\right)=V_{p} \sin (\theta) ; 0 \leq \theta \leq \pi \\ v=0 \\ \frac{\partial T}{\partial z}=0\end{array}\right.$

On the fixed plate $(\mathrm{z}=0,0<\mathrm{r}<\mathrm{R})$ :

$u=v=\frac{\partial T}{\partial z}=0$

On the symmetry axis $(\mathrm{r}=0,0<\mathrm{z}<\mathrm{L})$ :

$\frac{\partial T}{\partial r}=\frac{\partial u}{\partial r}=0$ and $v=0$

On the lateral wall:

- $\left(\mathrm{r}=\mathrm{R}, 0<\mathrm{z}<\mathrm{L}_{1}\right.$ and $\left.\mathrm{L}_{2}<\mathrm{Z}<\mathrm{L}\right)$ :

$u=v=\frac{\partial T}{\partial r}=0$

- $\left(\mathrm{r}=\mathrm{R}, \mathrm{L}_{1}<\mathrm{z}<\mathrm{L}_{2}\right)$ :

$\mathrm{u}=\mathrm{v}=0$ and $T=$ cte $=2 T_{0}$

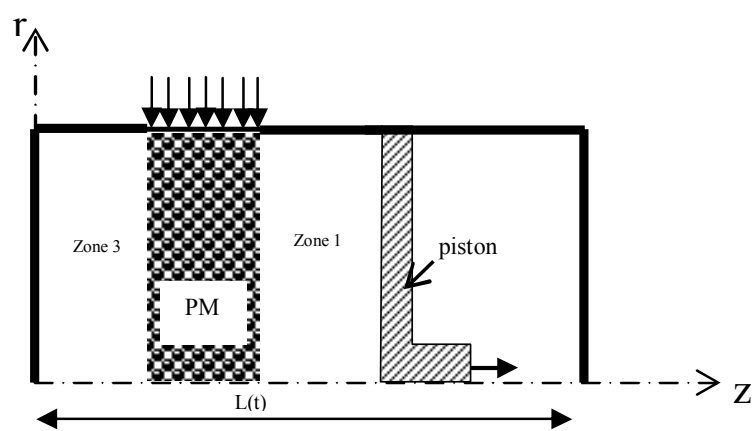

Fig. 1, Problem geometry.

The heat transfer coefficient in terms of the local Nusselt number for a pipe can be calculated as:

$N u(z, t)=\frac{\left.2 \frac{\partial T}{\partial r}\right|_{r=R}}{T(r=R)-T_{m}}$

The bulk temperature for flow in a pipe can be calculated as:

$T_{m}=\frac{\int_{0}^{R} u T r d r}{\int_{0}^{R} u r d r}$ 
The calculation of Mean Nusselt number is obtained via:

$$
N u(t)=\int_{L p} N u(z) d z
$$

The dimensionless convective heat flux at a cross section is defined by:

$$
\phi=\int_{0}^{R} \rho C_{p} u T 2 \pi r d r
$$

The instantaneous exchanged heat flux transferred through the porous insert is defined by:

$$
Q=\left.\phi\right|_{\text {exit of porous insert }}-\left.\phi\right|_{\text {entrance of porous insert }}
$$

\section{NUMERICAL METHOD AND SOlution Procedure}

The coupled differential equations for both fluid and porous regions are solved simultaneously along with the corresponding boundary conditions by using the control-volume-based finite element method of Baliga et al. (1980; 1983). The partial differential equations for all dependent variables are discretized by integrating the equations over the computational cells. In the derivation of algebraic approximations to surface integrals of convection and diffusion fluxes, the dependent variables $\mathrm{u}$ and $\mathrm{v}$ are interpolated linearly. The temperature is interpolated linearly in diffusion terms and by a Flow-Oriented upwind scheme (FLO) in convection terms.

The calculation domain is divided into two parts: a fixed part with a constant volume and a fixed uniform grid, appointing the zone of the cylinderhead and the porous zone, and a moved part deforming with the piston movement and having a time dependent volume and a moving grid. An extensive numerical experimentation was performed to establish grid independence results. It is found that an equally spaced grid mesh $241 * 131$ provides a grid independence results.

The coupled pressure-velocity equations together with the after mentioned boundary conditions are solved by the SIMPLER algorithm of Patankar et al. (1972) using an available discretization code. Starting from the given initial conditions, convergence is achieved after some iteration. The convergence criterion adopted was that relative variations in velocity and temperature between two successive iterations were smaller than $10^{-4}$. To obtain better convergence properties, the unsteady terms in these equations are implicitly treated. For each time step, the discretizated equations are solved using a line-by-line procedure. In order to validate the present numerical code, two computing cases are considered. In the first case, our numerical results were compared with a numerical data of a piston driven compressible flow with a sinusoïdal piston velocity (Duguet, 2004). The geometry consists of a vertical cylinder with a moving piston during the compression stroke. The parameters of resolution are defined by: $\mathrm{Re}=68, \mathrm{t}_{\mathrm{c}}=4.2$ and $\mathrm{L}(\mathrm{t}=0)=0.21$. As displayed in Fig. 2, the present numerical results are in fair agreement with the published one.

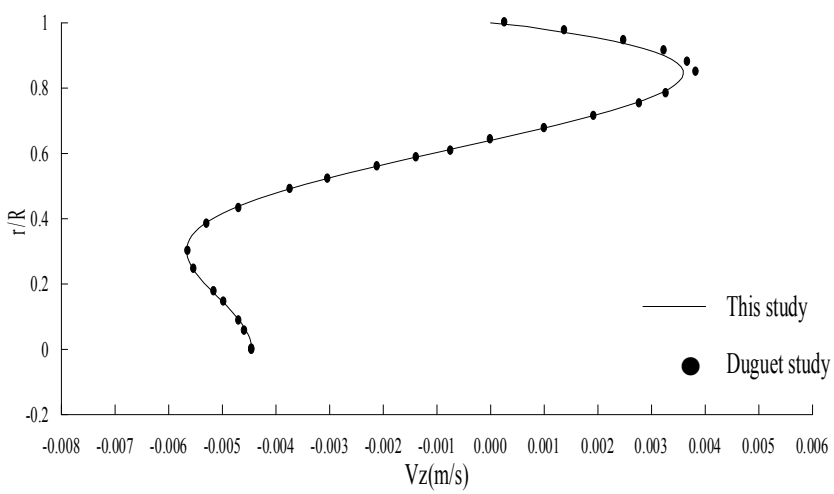

Fig. 2. Radial velocity profile, $U$ for the maximal piston velocity

The second test case concerns a problem of pulsating flow in a channel filled with porous media (Kim et al. 1994). In this case, satisfactory agreement was also obtained for the velocity profiles in Fig. 3.

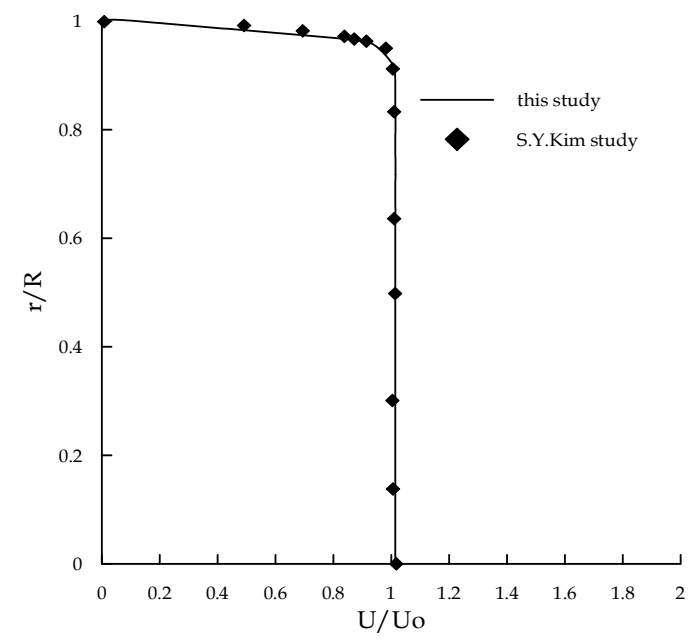

Fig. 3. Radial velocity profiles, $U$ in a channel filled with porous media for $\mathrm{Da}=10^{-4}$

\section{Numerical Results}

The character of piston-driven flow is marked by the non-dimensional parameter that is the Reynolds number based on the maximum piston velocity. In the case of a pipe partially filled with a porous medium, the Darcy number $\mathrm{Da}$, the ratio $R_{c}$ and the ratio $R_{k}$ are also essential ingredients for understanding the thermal and dynamic behavior. The present paper focuses on the influence of the above parameters on fluid flow and heat transfer in the pipe provided with a piston and partially filled with a porous insert. All cases simulated herein are compiled in Table 1.

The resulting computational fields were extracted at different moments to analyze sufficiently the structure of the fluid flow and heat transfer during expansion stroke. Figure $4 \mathrm{~b}$ displays these computational fields for a general case $\left(\mathrm{Re}=300, \mathrm{Da}=10^{-4}, \mathrm{R}_{\mathrm{c}}=10\right.$ and $\left.\mathrm{R}_{\mathrm{k}}=20\right)$. 

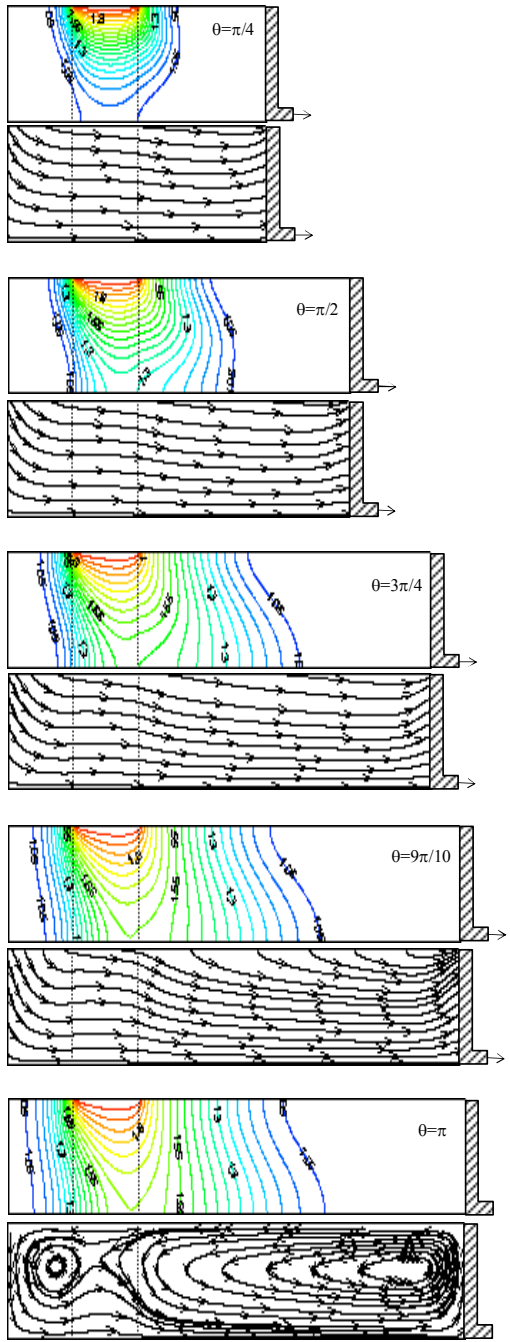

b
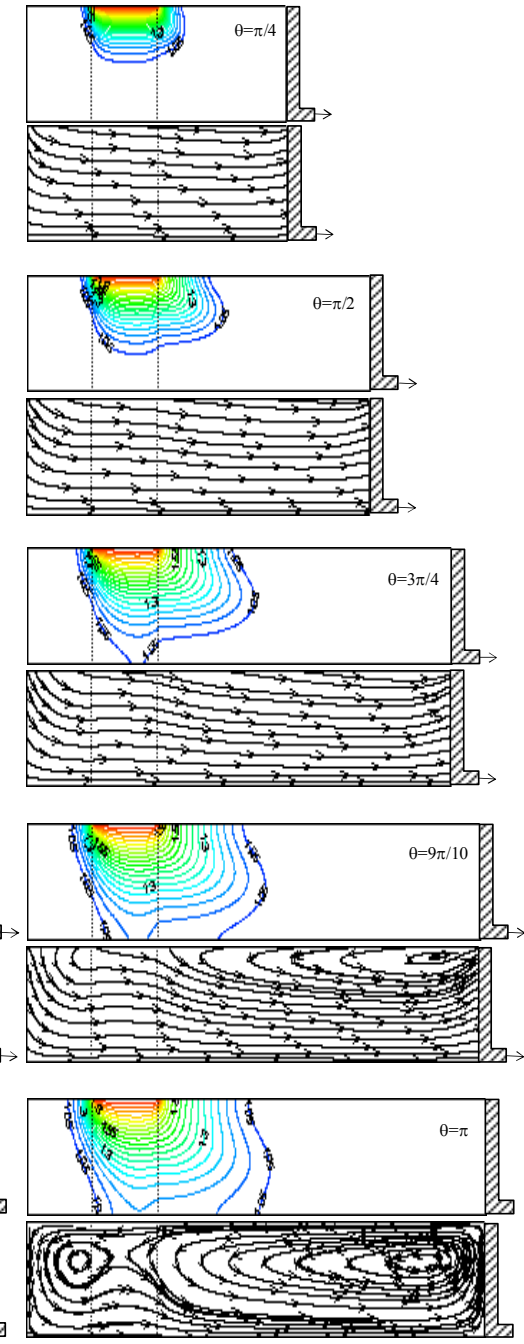

c
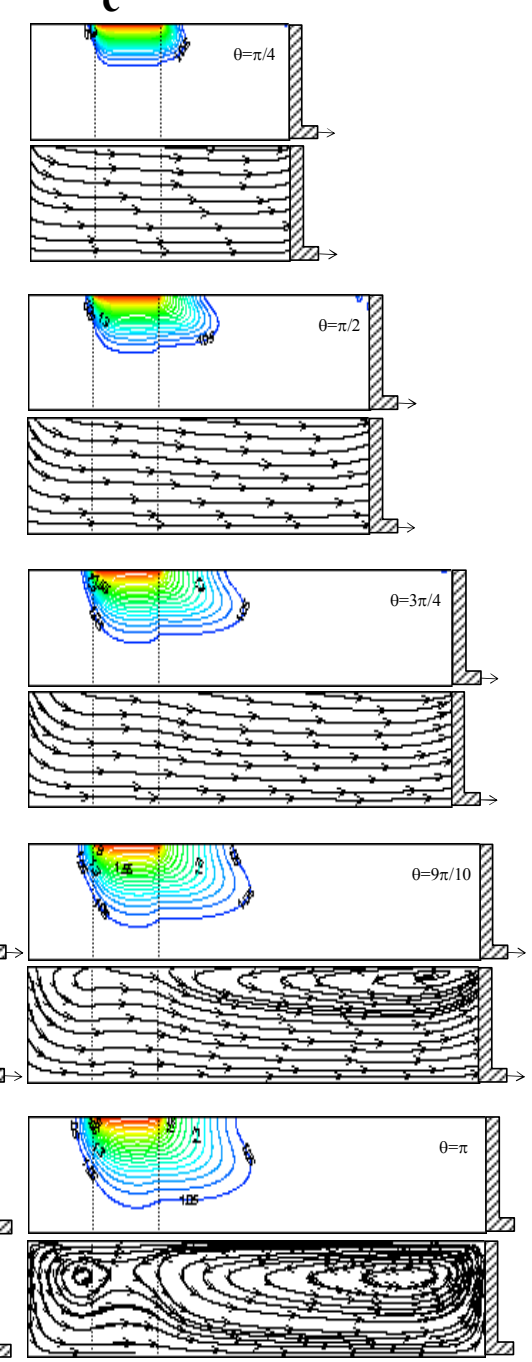

Fig. 4. Effect of Reynolds number on isotherms and streamlines during expansion stroke a: $R e=100-b: \operatorname{Re}=300-c: R e=500$

Table 1 Input data of governing parameters

\begin{tabular}{|c|c|c|c|c|c|c|}
\hline$\varepsilon$ & $\operatorname{Re}$ & Da & $\mathrm{R}_{\mathrm{k}}$ & $\mathrm{R}_{\mathrm{c}}$ & $\mathrm{P}_{\mathrm{r}}$ & $\mathrm{L}_{\mathrm{p}}$ \\
\hline \multicolumn{7}{|c|}{ Variation of Reynolds number, Re } \\
\hline 0.7 & 100 & $10^{-4}$ & 20 & 10 & 0.7 & $0.14 \mathrm{~L}(\theta=\pi)$ \\
\hline 0.7 & 300 & $10^{-4}$ & 20 & 10 & 0.7 & $0.14 \mathrm{~L}(\theta=\pi)$ \\
\hline 0.7 & 500 & $10^{-4}$ & 20 & 10 & 0.7 & $0.14 \mathrm{~L}(\theta=\pi)$ \\
\hline \multicolumn{7}{|c|}{ Variation of Darcy number, Da } \\
\hline 0.7 & 300 & $10^{-3}$ & 20 & 10 & 0.7 & $0.14 \mathrm{~L}(\theta=\pi)$ \\
\hline 0.7 & 300 & $10^{-4}$ & 20 & 10 & 0.7 & $0.14 \mathrm{~L}(\theta=\pi)$ \\
\hline 0.7 & 300 & $10^{-5}$ & 20 & 10 & 0.7 & $0.14 \mathrm{~L}(\theta=\pi)$ \\
\hline \multicolumn{7}{|c|}{ Variation of thermal conductivity ratio, $R_{k}$} \\
\hline 0.7 & 300 & $10^{-4}$ & 1 & 10 & $0.7^{\circ}$ & $0.14 \mathrm{~L}(\theta=\pi)$ \\
\hline 0.7 & 300 & $10^{-4}$ & 20 & 10 & 0.7 & $0.14 \mathrm{~L}(\theta=\pi)$ \\
\hline 0.7 & 300 & $10^{-4}$ & 200 & 10 & 0.7 & $0.14 \mathrm{~L}(\theta=\pi)$ \\
\hline \multicolumn{7}{|c|}{ Variation of heat capacity ratio, $R_{c}$} \\
\hline 0.7 & 300 & $10^{-4}$ & 20 & 1 & 0.7 & $0.14 \mathrm{~L}(\theta=\pi)$ \\
\hline 0.7 & 300 & $10^{-4}$ & 20 & 10 & 0.7 & $0.14 \mathrm{~L}(\theta=\pi)$ \\
\hline 0.7 & 300 & $10^{-4}$ & 20 & 100 & 0.7 & $0.14 \mathrm{~L}(\theta=\pi)$ \\
\hline
\end{tabular}

For very low values of $\theta(\theta \leq \pi / 4)$, no curved streamlines are detected in the entire domain due to the weak piston velocity and higher porosity value. At $\theta=3 \pi / 4$, the effect of the porous insert appears on the flow structure. First, the streamlines become curved near the lateral wall in the vicinity of the piston head and at the porous zone interfaces (inlet/exit). Later $(\theta=9 \pi / 10)$, a first recirculation zone appears at the piston head. At the same moment, a secondary weak recirculation zone emerges at the entrance of the porous insert. The appearance of the first vortex is due to the boundary layer which can separate from the wall under the action of the adverse pressure gradient. On the other hand, the presence of a higher porous medium creates a dissipative force taking place near the lateral wall. This force prevents the first recirculation zone from penetrating into porous zone from the exit interface. At the entrance interface of the porous insert, the convective forces create the stretching of the secondary recirculation zones axially toward the cylinder head and radially toward the symmetry axis. Later, the primary and the secondary vortexes, localized on both sides, are joined together in the porous region. At the end of the expansion stroke, the both clockwise recirculation zones dominate the flow field in the entire domain. Initially, the temperature is uniformly set to $\mathrm{T}_{0}$. During the expansion stroke, Fig. $4 \mathrm{~b}$ indicates the radial and axial heat transfer leading to the porous wall temperature condition. The heat transfer enhancement is the result of both convection and conduction.

Indeed, the heat transfer mechanism is much dominated by the transverse heat conduction in porous media layer 

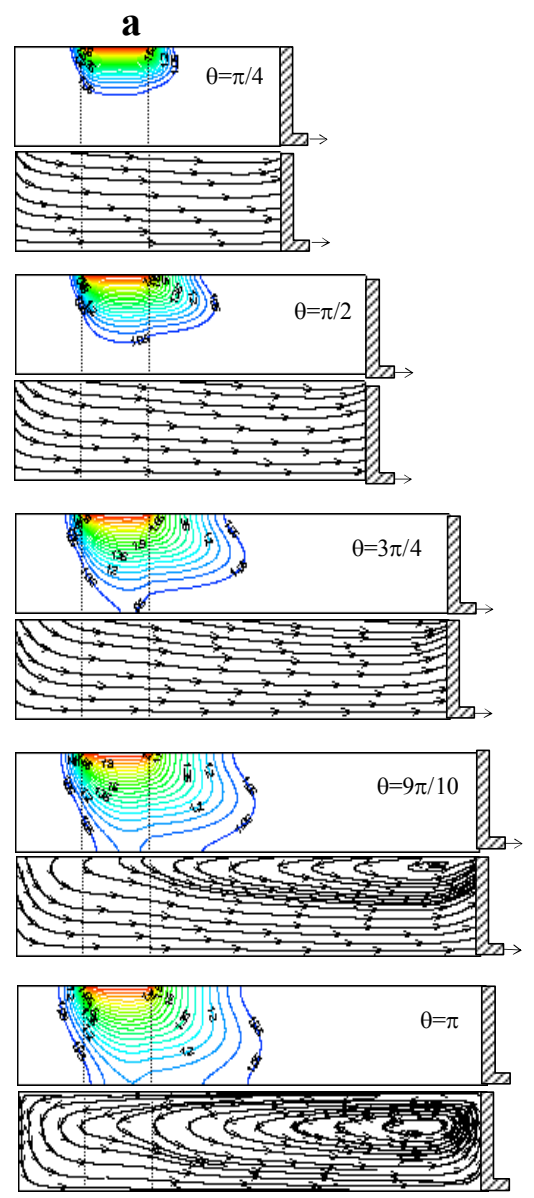

Fig. 5. Effect of Darcy number on isotherms and streamlines during expansion stroke
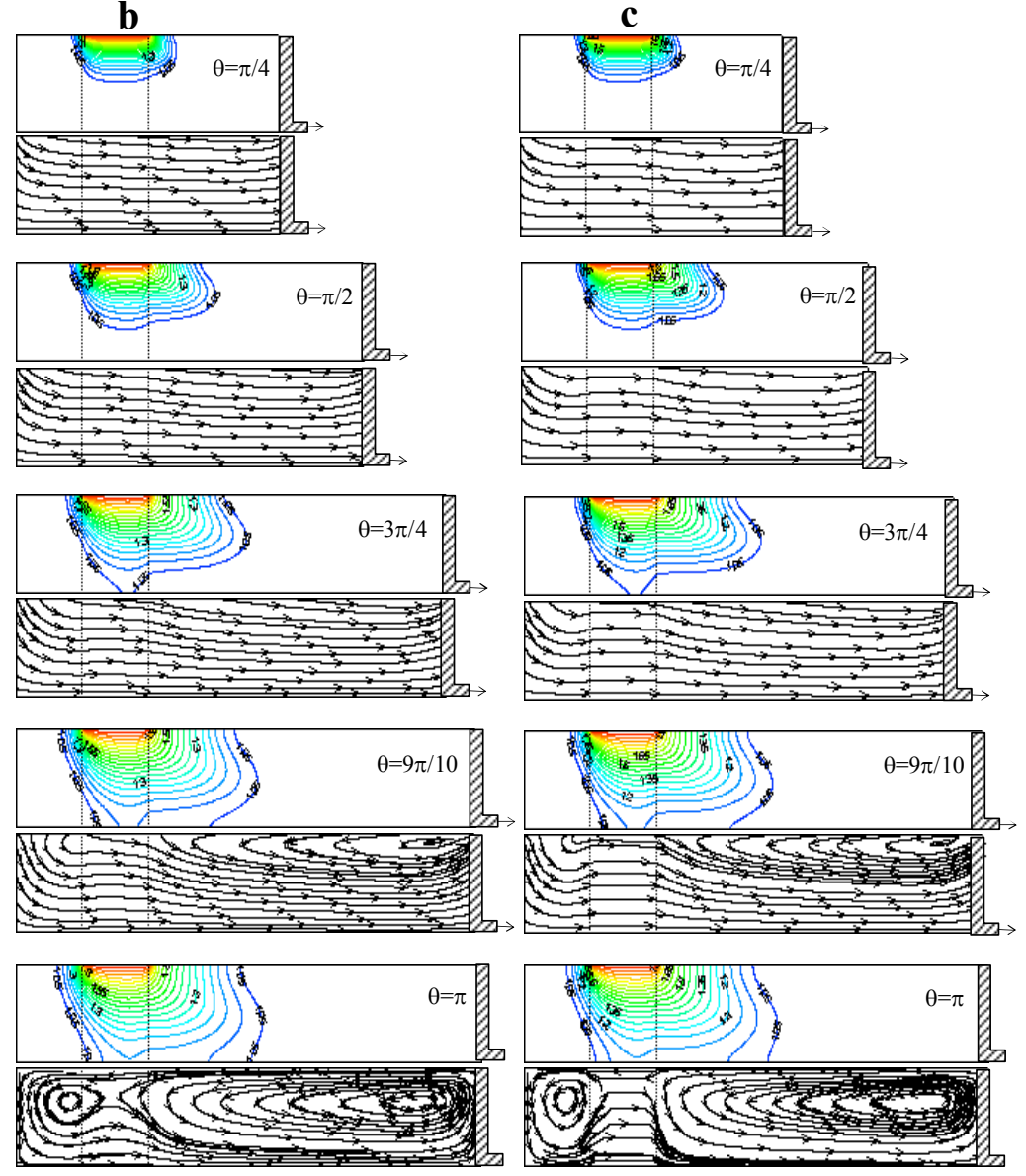

a: $\mathrm{Da}=10^{-3}-\mathrm{b}: \mathrm{Da}=10^{-4}-\mathrm{c}: \mathrm{Da}=10^{-5}$

and by the axial heat convection in the expanded volume. It is worth noting that the observed isotherms are slightly distorted along the entrance and especially the exit porous interfaces. That gives rise to the creation of a temperature gradient in the corresponding areas therefore the creation of a thermal heat flux.

\subsection{Effect of Reynolds Number}

Flow patterns and temperature fields for two different values of Reynolds number $(\mathrm{Re}=200$ and 500) are plotted in Fig. 4. The increase of the Reynolds number causes a faster evolution of the flow structure. Hence, after its apparition, the second pair of vortexes penetrates the porous zone. Indeed, the dissipative forces in the porous insert are weakened by the convective forces which are proportional to the Reynolds number. On the other hand, it is observed that the effect of Reynolds number on heat transfer is fairly noticeable. In fact, for lower Reynolds number, the heat transfer mechanism is dominated by heat conduction in porous media layer especially during the acceleration phase $(\theta \leq \pi / 2)$. During the deceleration phase $(\theta \geq \pi / 2)$, the recirculation zone causes an amplification of the convective heat transfer in the first zone toward the piston. When the Reynolds number is increased, the colder flow penetrates the porous region (in the inlet) very rapidly and the convective heat flux dominates the heat transfer. Also, it is worth noting that the isotherms are more deviated and distorted toward the piston head when Re increases. The axially heat transfer is more important than the radial one. This makes the first zone less heated when decreasing the values of Re.

\subsection{Effect of Darcy Number}

The effect of the Darcy number on the streamlines and isotherms is depicted in Fig. 5. The Darcy number $\left(10^{-5} \div 10^{-3}\right)$ is directly proportional to the permeability of the porous medium.

For small values of the Darcy numbers, the porous matrix appears like an obstacle. This makes the fluid particles moving with a week kinetic energy in this region. On the other hand the flow in both sides of the porous region is almost independent. Consequently, the vortexes appearance and their radial and axial stretching are more accelerated in the fluid regions (zones 1 and $3)$. At the end of the expansion stroke, both recirculation zones are not joined and dominate the flow field in each fluid region. The increase of the Darcy numbers makes the flow structure more homogenous in the entire domain by reducing the effect of the porous insert. In fact, in this case only the piston head vortex appears and presents an axial and radial stretching compared to the case of a cylinder without a porous insert.

The temperature field is weak affected by the $\mathrm{Da}$ number. In fact, the isotherm patterns indicate the 

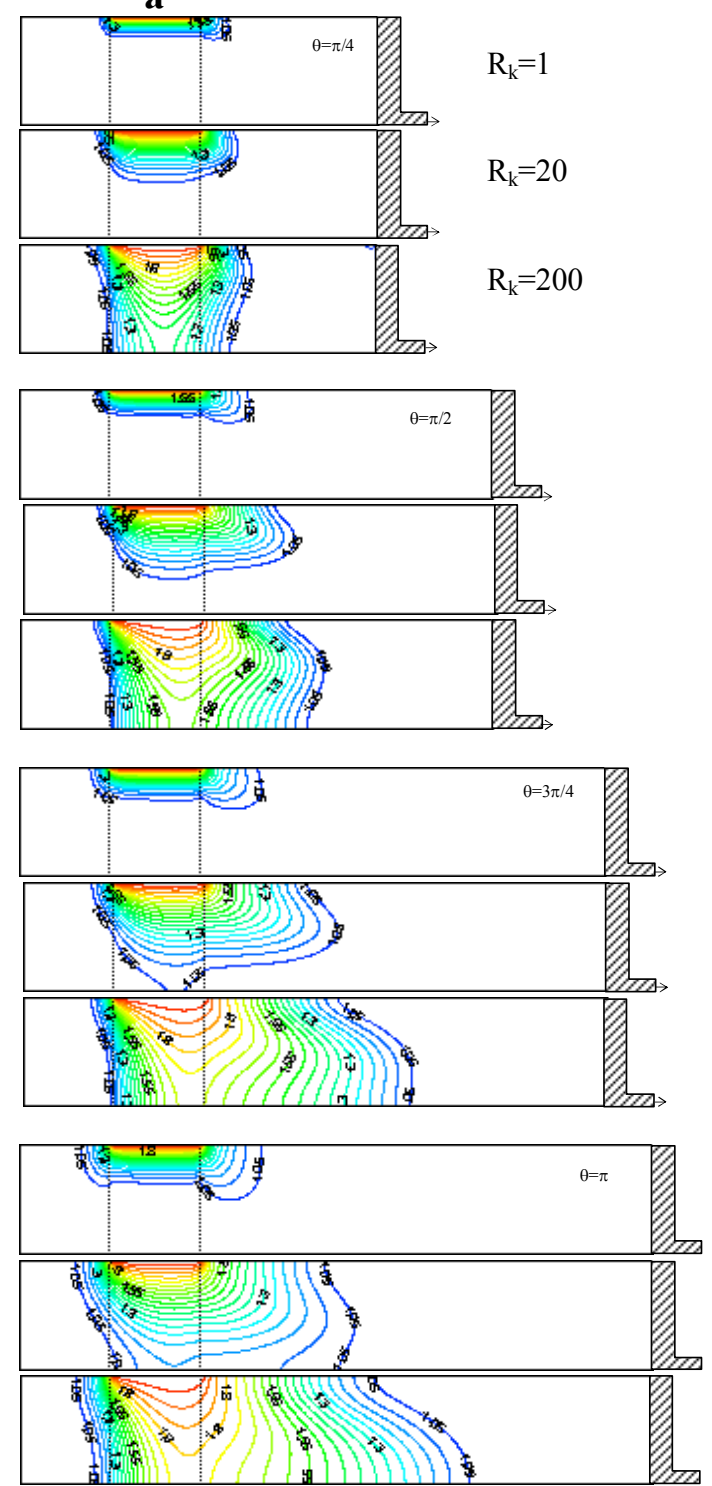

b
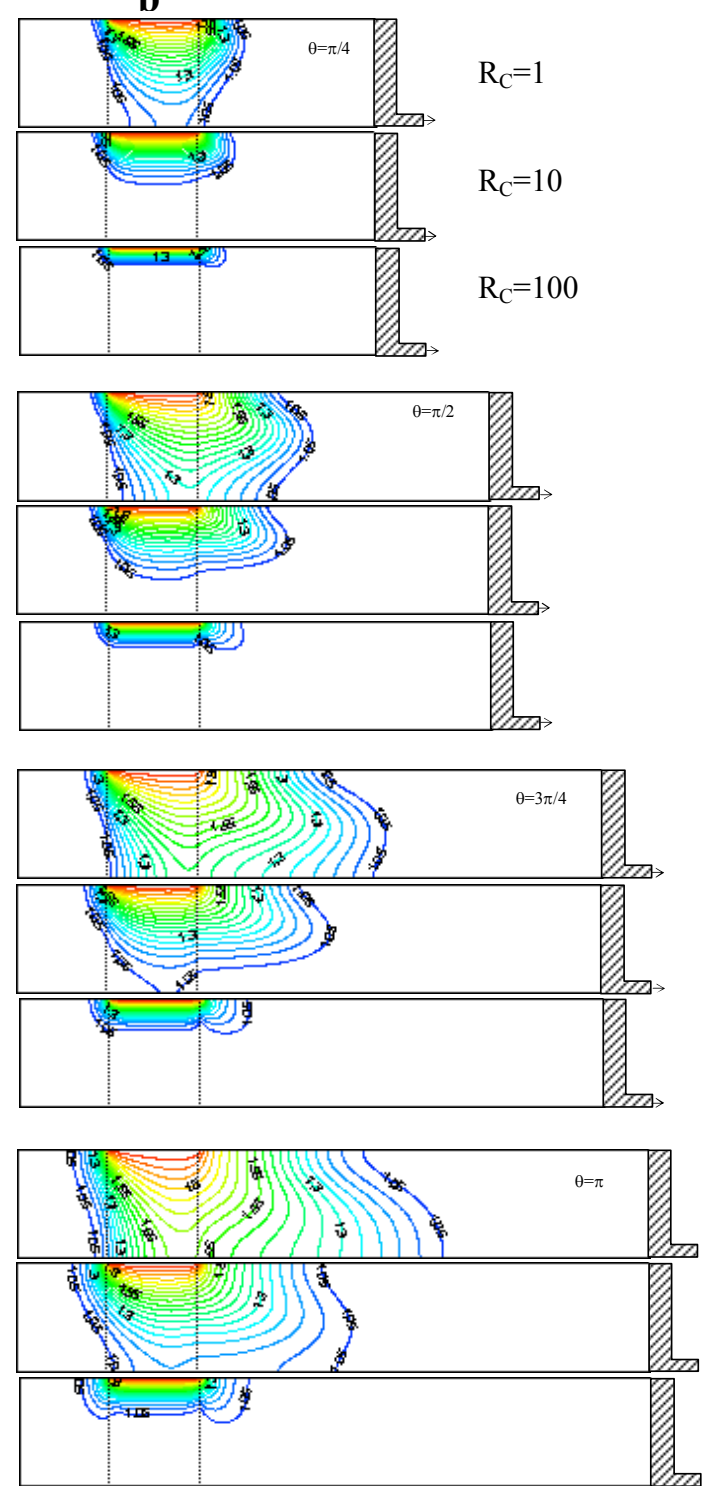

Fig. 6. Effect of thermal porous properties on isotherms during expansion stroke a: Thermal conductivity ratio- $b$ : Heat capacity ratio

conductive nature of heat transfer which is essentially in the r-direction. This trend is due to the significant imposed thermal conductivity ratio $\left(\mathrm{R}_{\mathrm{k}}=20\right)$.

\subsection{Effect of Thermal Porous Insert Properties}

The effect of the porous insert thermophysical properties $R_{k}$ and $R_{c}$, are presented in Fig. 6. Obviously, using porous insert with higher thermal conductivity and also with lower heat capacity, improves the radial conductive heat transfer from the heated lateral wall to the symmetry axis. This enhances also the axial convective heat transfer towards the piston region.

\subsection{Convective Heat Flux}

The quantity $(\mathrm{Q})$ is of great interest in this application to quantify the total heat flux convected during the piston stroke through the porous insert. It can be seen that the evolution of the instantaneous exchanged heat flux through porous insert adopts sinusoidal form like the fluid and piston velocities. As expected, the exchanged convective heat flux (Q) increases considerably with the Reynolds number (Fig.7a). In fact, the increase of the Reynolds number implies the increase of the velocity of the fluid particles which exchange a lower heat quantity through the solid porous matrix. Thus the thermal boundary layer thickness becomes thinner with the increase of Re (Zhao and Cheng, 1995). However, when the porous matrix is characterized with a higher thermal conductivity and lower heat capacity, the convective heat flux becomes important (Fig.7c, 7d). The effect of the Darcy number is not predictable as the effect of Re, $R_{k}$ and $R_{c}$ on the instantaneous convective heat flux (Fig. 7b). This is due to the higher thermal conductivity of the porous insert. However, we can note that by increasing the permeability, the convective heat flux decreases essentially when the effect of the thermal conductivity ratio is excluded $\left(\mathrm{R}_{\mathrm{k}}=1\right)$. 


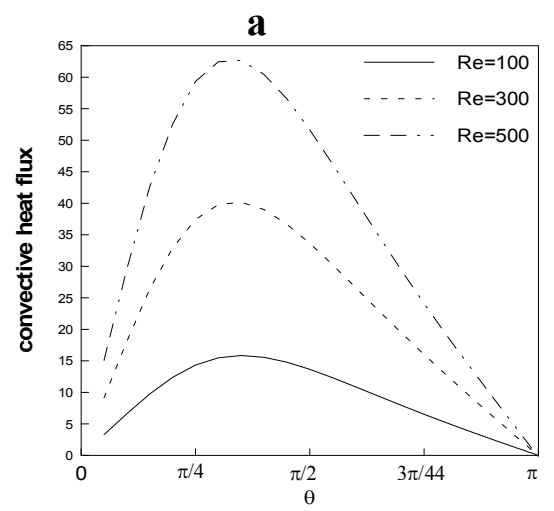

b
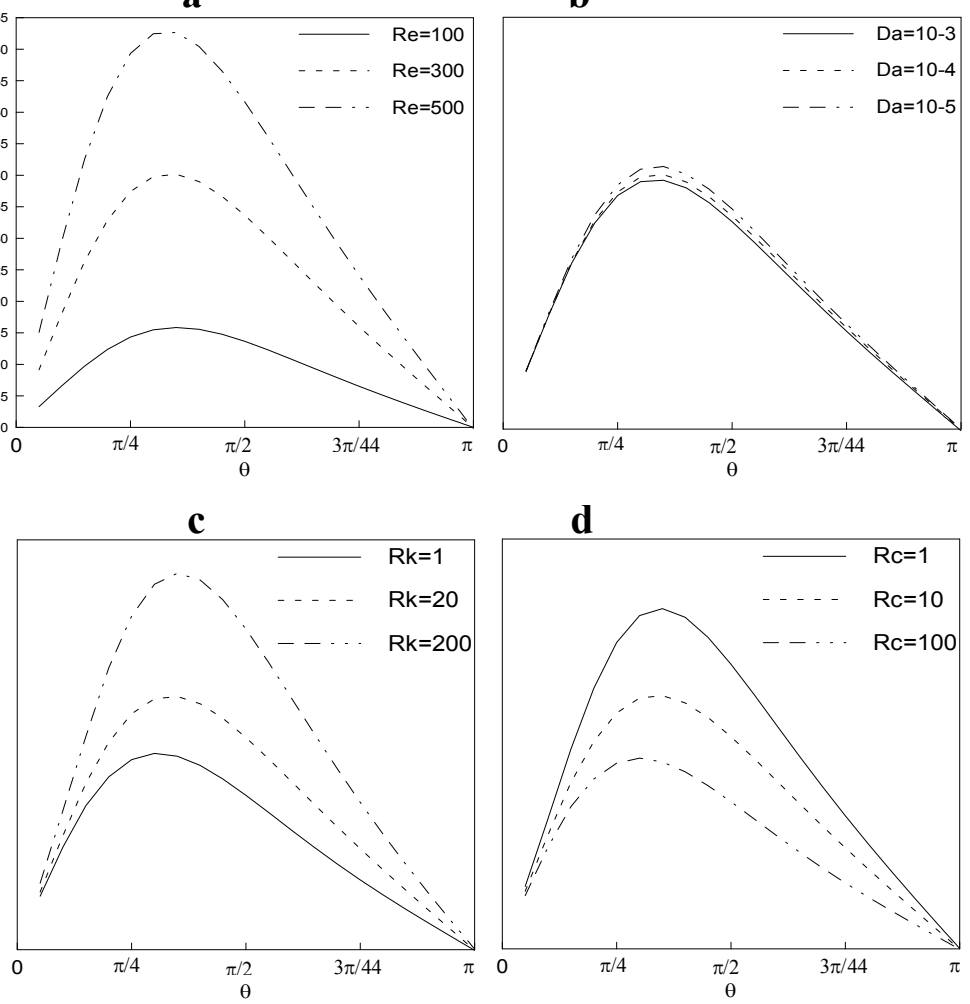

d

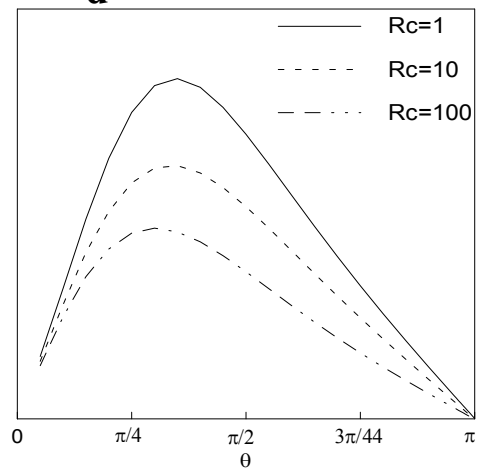

Fig. 7. Convective heat flux evolution during expansion stroke for different: a: Reynolds number- b: Darcy number- c: Thermal conductivity ratio- d: Heat capacity ratio
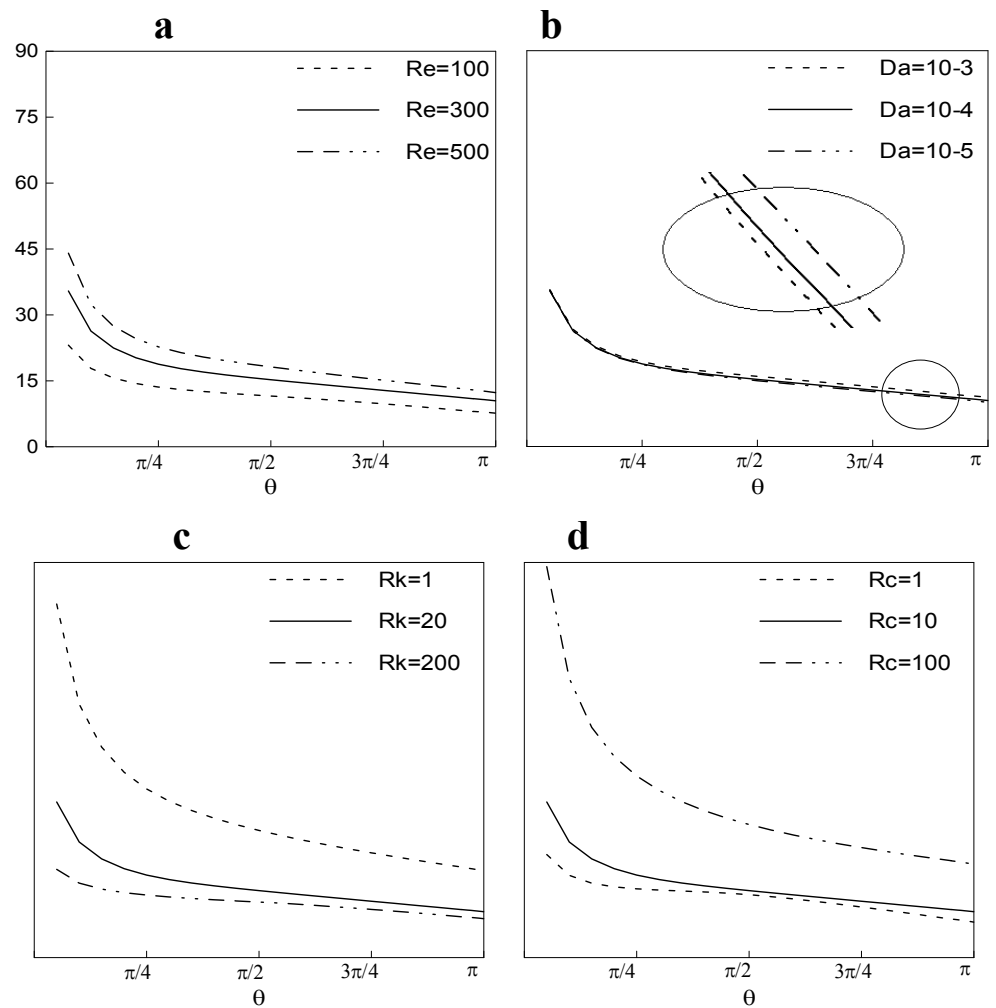

Fig. 8. Mean Nusselt number evolution during expansion stroke for different: a: Reynolds number- $b$ : Darcy number- $c$ : Thermal conductivity ratio- d: Heat capacity ratio 


\subsection{Nusselt Number}

According to Fig. 8, the overall patterns of the mean Nusselt number are qualitatively similar. Indeed these patterns presents a phase of great decrease, during first half of the acceleration phase $(\theta<\pi / 4)$, followed by a weak decrease phase $(\pi / 4<\theta<\pi / 2)$ and finally a quasi static mode during the deceleration phase. It is of interest to note that because of the great thermal conductivity of the porous insert $\left(\mathrm{R}_{\mathrm{k}}=20\right)$, the difference between the heating temperature and that of the porous zone, on the lateral wall zone, is small. This explains the high values of the mean Nusselt number directly related to the temperature gradient in this zone. Quantitatively, the mean Nusselt number increases by increasing the Reynolds number and the heat capacity ratio and by decreasing the Darcy number and the thermal conductivity ratio.

\section{Conclusion}

Detailed numerical analyses were performed to characterize the piston driven flow and the heat transfer in a pipe partially filled with a porous insert during an expansion stroke. The main emphasis was placed on the fluid structure and the heat transfer enhancement by a porous media insertion. This enhancement was explored by various pertinent dimensionless parameters such as the Reynolds number, the Darcy number, the thermal conductivity and the heat capacity ratios.

The results of this investigation illustrate that the Reynolds and the Darcy numbers have a significant effect on flow structure evolution. In fact, the increase of the Reynolds number and the decrease of the Darcy number accelerate the flow evolution and enhance the conductive heat transfer in the r-direction towards the symmetry axis and the convective heat transfer axially towards the piston. The higher heat transfer enhancement was observed by increasing the heat capacity ratio and decreasing the thermal conductivity ratio with a great Reynolds number and a low Darcy number.

\section{REFERENCES}

Baliga, B.R. and S.V. Patankar (1980). A new finiteelement formulation for convection-diffusion problems. Numerical Heat Transfer 3, 393-409.

Baliga, B.R. and S.V. Patankar (1983). A controlvolume finite element method for two-dimensional fluid flow and heat transfer. Numerical Heat Transfer 6, 245-261.

Boucher, J., F. Lanzetta and P. Nika (2007). Optimization of a dual free piston Stirling engine. Applied Thermal Engineering 27(4), 802-811.

Dhahri, H., A. Boughamoura and S.B. Nasrallah (2006). Numerical study of heat transfer in a porous pipe subjected to reciprocating flow. Journal of Porous Media 9(4), 1-17.

Duguet, Y. (2004). Simulation Numérique de l'instabilité Hydrodynamique dans un Cylindre de Gaz Tournant Soumis à une Compression
Périodique. Thèse de Doctorat, Ecole centrale de Lyon.

Ergun, S. (1952). Fluid flow through packed columns. Chemical Engineering Progress 48, 89-94.

Fu, W.S., H. C. Huang and W.Y. Liou (1996). Thermal enhancement in laminar channel flow with a porous block. International Journal of Heat and Mass Transfer 39, 2165-2175.

Hong, Y.J., S.J. Park, H.B. Kim and D.Y. Koh (2002). Dynamic analysis of a free piston Stirling refrigerator. Cryocoolers 12, 103-108.

Kim, S.Y., B.H. Kang and J.M. Hyun (1994). Heat transfer from pulsating flow in a channel filled with porous media. International Journal of Heat and Mass Transfer 37, 2025-2033.

Nika, P., F. Lanzetta, J. Boucher, E. Gavignet and J.E. Rakotoniaina (2004). Aspects dynamiques et thermiques de l'ecoulement oscillant dans la matrice poreuse d'un régénérateur de machine Stirling. Congrès Français de Thermique- SFT Giens, 825-830.

Park, C.W. and M. Kaviany (2002). Evaporationcombustion affected by in cylinder, reciprocating porous regenerator. Journal of Heat Transfer 124 (1), 184-194.

Patankar, S.V. and D.B. Spalding (1972). A calculation procedure for heat, mass and momentum transfer in three-dimensional parabolic flows. International Journal of Heat and Mass Transfer 15, 17871806.

Vafai, K. (1984). Convective flow and heat transfer in variable-porosity media. Journal of Fluid Mechanics 147, 233-259.

Vafai, K. and S.J. Kim (1990). Analysis of surface enhancement by a porous substrate. Journal Heat Transfer 112 (3), 700-706.

Zahi, N., A. Boughamoura, H. Dhahri and S.B. Nasrallah (2008). Flow and heat transfer in a cylinder with a porous medium insert along the compression stroke. Journal of Porous Media 11(6), 525-540.

Zhao, T.S. and P. Cheng (1995). A numerical solution of laminar forced convection in a heated pipe subjected to a reciprocating flow. International Journal of Heat and Mass Transfer 38, 30113022. 